\title{
The Role of the Central Nucleus of the Amygdala in Mediating Fear and Anxiety in the Primate
}

\author{
Ned H. Kalin, ${ }^{1,2}$ Steven E. Shelton, ${ }^{1}$ and Richard J. Davidson ${ }^{1,2}$ \\ Departments of ${ }^{1}$ Psychiatry and ${ }^{2}$ Psychology, University of Wisconsin, Madison, Wisconsin 53719
}

\begin{abstract}
Numerous studies demonstrate that the rhesus monkey is an excellent species with which to investigate mechanisms underlying human emotion and psychopathology. To examine the role of the central nucleus of the amygdala ( $\mathrm{CeA}$ ) in mediating the behavioral and physiological responses associated with fear and anxiety, we used rhesus monkeys to assess the effects of excitotoxic lesions of the CeA. Behavioral and physiological responses of nine monkeys with bilateral CeA destruction (ranging from 46 to $98 \%$ ) were compared with five animals with asymmetric lesions ( $42-86.5 \%$ destruction on the most affected side) and with 16 unoperated controls. Results suggest that similar to rodent species, the primate CeA plays a role in mediating fear- and anxiety-related behavioral and endocrine responses. Compared with controls and the asymmetric-lesion group, bilaterally lesioned monkeys displayed significantly less fear-related behavior when exposed to a snake and less freezing behavior when confronted by a human intruder. In addition, bilaterally lesioned monkeys had decreased levels of CSF corticotrophin-releasing factor (CRF), and both lesioned groups had decreased plasma ACTH concentrations. In contrast to these findings, patterns of asymmetric frontal brain electrical activity, as assessed by regional scalp EEG, did not significantly differ between control and lesioned monkeys. These findings suggest that in primates, the CeA is involved in mediating fear- and anxiety-related behavioral and pituitary-adrenal responses as well as in modulating brain CRF activity.
\end{abstract}

Key words: amygdala; anxiety; corticotropin; fear; primates; central nucleus

\section{Introduction}

Rhesus monkeys are an excellent species with which to investigate mechanisms relevant to human emotion and psychopathology. Rhesus monkeys and humans share similarities in social and emotional behavior, and rhesus monkeys express psychopathology similar to that observed in humans (Kalin, 1993; Bakshi and Kalin, 2000). Importantly, both species have a well developed prefrontal cortex that is highly and reciprocally interconnected with the amygdala (Amaral et al., 1992; Fuster, 1997), and evidence suggests that these structures are an important part of the circuitry involved in regulation of emotion in humans (Davidson et al., 2000). For these reasons, we have been using rhesus monkeys to characterize fear- and anxiety-related behavior and physiological responses and to elucidate the brain mechanisms underlying these responses.

Numerous studies in rodents, primates, and humans demonstrate involvement of the amygdala in mediating the emotional, behavioral, and physiological responses associated with fear and anxiety (Blanchard and Blanchard, 1972; Aggleton and Passingham, 1981; Meunier et al., 1999; Davis, 2000; LeDoux, 2000; Davis and Whalen, 2001; Kalin et al., 2001; Amaral, 2002; David-

Received Jan. 26, 2004; revised April 29, 2004; accepted May 3, 2004.

This work was supported by The HealthEmotions Research Institute and Meriter Hospital Grants MH46729, MH52354, and MH61083. We thank H. Van Valkenberg, K. Lee, K. Tietz, J. Droster, M. Grimes, T. Johnson, J. King, and the staff at the Harlow Center for Biological Psychology and the National Primate Research Center at the University of Wisconsin for their technical support and Dr. David Amaral for providing his expertise in defining the CeA.

Correspondence should be addressed to Ned H. Kalin, University of Wisconsin, Department of Psychiatry, 6001 Research Park Boulevard, Madison, WI 53719. E-mail: nkalin@wisc.edu.

DOI:10.1523/JNEUROSCI.0292-04.2004

Copyright $\odot 2004$ Society for Neuroscience $\quad$ 0270-6474/04/245506-10\$15.00/0 son, 2002). In addition, altered amygdala activity has been implicated in some types of psychopathology (Drevets and Raichle, 1995; Davidson, 2002; Schwartz et al., 2003a). The amygdala is a complex structure consisting of numerous subnuclei (Amaral et al., 1992). Within the amygdala, the central nucleus of the amygdala $(\mathrm{CeA})$ is in a strategic position to mediate many aspects of fear and anxiety, because CeA neurons project to sites involved in mediating different aspects of the stress response, including the hypothalamus, basal forebrain, and brainstem (Amaral et al., 1992; Davis, 1992). The CeA also receives input from many of these structures (Amaral et al., 1992). In contrast to the CeA, the basolateral regions of the amygdala are bidirectionally connected to the cortex, including orbitofrontal and ventromedial regions (Amaral et al., 1992). Extensive studies in rodents have examined the functional contributions of specific amygdala nuclei and demonstrate a role of the CeA in mediating various behavioral and physiological responses elicited by exposure to conditioned and unconditioned stimuli (Davis, 1992). However, very few studies have been performed in primates that have attempted to parse the functions of the amygdala subnuclei (Aggleton and Passingham, 1981). Until recently, amygdala lesion studies in nonhuman primates that have assessed effects on emotion have used large nonselective lesions (Kluver and Bucy, 1939) or methods that have destroyed neurons as well as fibers of passage (Weiskrantz, 1956; Aggleton and Passingham, 1981; ZolaMorgan et al., 1991; Kling and Brothers, 1992). More recent studies using excitotoxic lesions demonstrate a more subtle role of the primate amygdala in mediating emotion, learning, and behavior (Murray and Mishkin, 1998; Meunier et al., 1999; Emery et al., 2001; Kalin et al., 2001). 
In previous work, we described the adaptive behavioral and physiological responses that are associated with fear and anxiety in rhesus monkeys (Kalin, 1993; Kalin et al., 1998). We found that dispositionally anxious monkeys not only engage in more intense anxiety-like behavior but also have increased basal plasma levels of the stress hormone cortisol (Kalin et al., 1998), increased CSF concentrations of the anxiogenic peptide CRF (Kalin et al., 2000), and extreme asymmetric right frontal brain electrical activity as assessed by EEG (Kalin et al., 1998). We suggested that when extreme, these characteristics define an anxious endophenotype, which we propose is analogous to the description of extremely shy and inhibited children that are at risk to develop anxiety disorders (Kagan et al., 1988; Kalin and Shelton, 1989; Biederman et al., 1993; Schwartz et al., 2003b). In an initial study in rhesus monkeys, we assessed the effects of large ibotenic acid lesions of the amygdala on the behavioral and physiological parameters relevant to the anxious endophenotype (Kalin et al., 2001). The current study extends our previous work by making small selective lesions in the CeA region of adolescent monkeys to examine the extent to which the CeA mediates many of these responses. We focused on adolescence, because in humans it is a period of emergence of affective and anxiety disorders and our ultimate goal is to provide insight into the developmental issues related to the onset of these disorders.

\section{Materials and Methods}

Experimental subjects. Rhesus monkeys (Macaca mulatta) were used as experimental subjects. The animals were housed at the Harlow Primate Laboratory and at the Wisconsin National Primate Research Center. Animal housing and experimental procedures were in accordance with institutional guidelines. Eighteen males underwent lesioning procedures at an average age of 34.9 months. Sixteen unoperated male controls were used for comparison and at the beginning of the study were on average 34.6 months of age.

Central nucleus amygdala lesions. Because of the large variability in skull and brain size in rhesus monkeys, attempts to produce stereotaxic lesions in monkeys with no guidance other than standard atlases were subject to substantial inaccuracy. The method for producing CeA lesions was adapted from methods described previously (Amaral and Price, 1983; Murray and Mishkin, 1998). All surgical procedures were performed under strict aseptic conditions and deep anesthesia. Landmarks were established by stereotactically implanting two $3 \mathrm{~mm}$ glass beads filled with a $3 \%$ solution of copper sulfate [which is hyperintense in T1 (timing one)-weighted magnetic resonance imagings (MRIs)] into shallow indentations in the skull. The beads were placed 8 and $12 \mathrm{~mm}$ lateral to the midline and 12 and $16 \mathrm{~mm}$ anterior to the intra-aural line. This placed them in an area above the amygdala. The beads were held in place with dental acrylic. At least $2 \mathrm{~d}$ after the beads were implanted, the subject was brought to the magnetic resonance (MR) imager. Under anesthesia (15 mg/kg ketamine and $0.1 \mathrm{mg} / \mathrm{kg}$ xylazine, i.m.), the monkey was placed in a plastic replica of a David Kopf Instruments (Tujunga, CA) stereotaxic apparatus that was positioned in the head coil. Using a 1.5 tesla GE Signa (Waukesha, WI) scanner, the brain was initially imaged in the coronal plane to verify its symmetric alignment to the stereotaxic apparatus and the scanner. The animal was then scanned in the sagittal plane to show the relationship of the beads to each coronal slice. For the coronal scan, a three-dimensional (3D) fast-spoiled gradient pulse (FSPG) sequence (3D/FSPGR/20) was used with a repetition rate of 9.4, a fractional echo time of $2.1 /$ fractional, one echo, a receiver bandwidth of $15.6 \mathrm{kHz}$, an inversion time of 400 , and a $20 \times 15$ field of view, using $256 \times 224 / 4$ excitations to create 60 contiguous $1 \mathrm{~mm}$ coronal brain images.

Measurements of the amygdala were made by laying a $1 \mathrm{~mm}$ matrix over the coronal MR image and centering it on the midline of the brain. The mediolateral, dorsoventral, and anteroposterior location of the CeA in relation to the bead was determined from the MR images. These mea- surements were used to plan the stereotactic coordinates for the ibotenic acid injections.

Animals were returned to the surgical suite for the lesioning procedure. Prophylactic doses of the antibiotic cefazolin $(20 \mathrm{mg} / \mathrm{kg}$, i.m.) were given before surgery. The animals were preanesthetized with ketamine $\mathrm{HCl}(10 \mathrm{mg} / \mathrm{kg})$, fitted with an endotracheal tube, and placed on isofluorane gas anesthesia. Heart rate, electrocardiogram, $\mathrm{SPO}_{2}$, rectal temperature, $\mathrm{SPCO}_{2}$, and respiration were monitored throughout the surgical procedure. Using standard aseptic surgical techniques, the animals were mounted in a stereotaxic apparatus (David Kopf Instruments). The skull was exposed, and the skull opening was made above the intended lesion site as determined from the MRI procedure.

Between 2 and 10 injections of $1 \mu$ libotenic acid ( $1 \mathrm{mg}$ of ibotenic acid hydrate/100 $\mu$ l of PBS) were made to lesion the CeA. Ibotenic acid is a neurotoxin derived from Amanita (BioSearch Technologies, San Raphael, CA). Injections were made simultaneously bilaterally using a $10 \mu \mathrm{l}$ syringe (Hamilton, Reno, NV) at a rate of $0.2 \mu \mathrm{l} / \mathrm{min}$. A similar technique has been used previously to completely eliminate the amygdaloid complex while producing little damage to surrounding structures (Kalin et al., 2001). To control brain swelling, mannitol (1.5-2.0 gm/ $\mathrm{kg}$ ) was administered intravenously over 30 min during surgery.

After the ibotenic acid injections were made, the midline wound was sutured and the animal recovered from anesthesia. To ease postsurgical discomfort, a $0.01 \mathrm{mg} / \mathrm{kg}$ intramuscular dose of buprenorphine was given. After surgery, a $2 \mathrm{mg} / \mathrm{kg}$ intramuscular injection of dexamethasone was given as needed to control brain swelling. Antibiotics, steroids, and analgesics were administered after surgery as needed.

Lesion verification. Lesioned animals were humanely killed using methods consistent with the recommendations of the Panel on Euthanasia of the American Veterinary Medical Association. An overdose of pentobarbital was given intravenously, and the animals were perfused with heparinized PBS followed by $4 \%$ paraformaldehyde. The brains were removed and histologically processed. The brain slab containing the volume of interest was cryoprotected in 2\% DMSO and 20\% glycerol for 12-18 hr, encased in a gelatin matrix after hardening in $10 \%$ buffered formalin, and then freeze-sectioned at $40 \mu \mathrm{m}$. Every sixth section $(240$ $\mu \mathrm{m})$ was collected in standard phosphate-buffered $10 \%$ formalin. These sections were mounted on $2 \times 3$ inch glass slides, air dried, and stained for Nissl substance with thionine. To assess lesion-induced glial infiltration and to help define the boundaries of the CeA lesion, a matched set of coronal slices was stained with glial fibrillary acidic protein (GFAP). From an unoperated animal, Dr. David Amaral (University of California Davis, Davis, CA), a leading expert in macaque amygdala anatomy, defined the boundaries of the CeA in the 14 consecutive sections containing the CeA (Amaral et al., 1992). Figure $1 b$ shows 2 of the 14 coronal slices defining the boundaries of the CeA. The 14 sections throughout the extent of the CeA were used as templates on which the damage observed in matched sections from each lesioned animal was superimposed. Using $\mathrm{NIH}$ Image 1.63, the pixels shared by the lesion and the control CeA template were used to calculate the percentage of CeA destruction. To assess damage collateral to the CeA, sections were examined anteriorly and posteriorly to the region containing the CeA throughout the temporal lobe. On the basis of this examination, the amount of damage to the medial amygdala nuclei (medial and cortical nuclei); lateral, basal, and accessory basal nuclei; and basal forebrain (substantia innominata and nucleus basalis) was estimated.

Threat-related anxiety and defensive behaviors. To assess threat-related anxiety and different defensive behaviors, animals were first placed alone in an isolated cage, $79 \mathrm{~cm}$ wide $\times 76 \mathrm{~cm}$ high $\times 71 \mathrm{~cm}$ deep. The alone (A) condition lasted for the first 9 min during which the animal was placed in a cage by itself. During the next 9 min, a human entered the room and presented her profile to the animal, standing $2.5 \mathrm{~m}$ from the cage while avoiding any eye contact with the animal [no eye contact (NEC) condition]. The human then left the room for $3 \mathrm{~min}$, reentered the room, and for 9 min remained motionless $2.5 \mathrm{~m}$ from the cage while staring, with a neutral face, directly at the animal [stare (ST) condition]. Behavior and vocalizations were recorded on videotape (Kalin and Shelton, 1989).

The behavioral ratings were encoded into the computer from video- 
tapes by an experienced rater who was unaware of the treatment conditions. Using a computer-based behavioral scoring system, operationally defined behavior categories were entered as they occurred. Frequency and duration of individual behaviors were then calculated (Kalin et al., 2001). Definitions of the key behaviors are as follows: locomotion, ambulation of one or more full steps at any speed; freezing, a period of at least $3 \mathrm{sec}$ characterized by tense body posture without vocalizations and movement, other than slow movements of the head; experimenter orient, any nonhostile orienting behavior to the experimenter; experimenter hostility, any hostile behaviors directed at the intruder, such as barking, head bobbing, and ear flapping; coo, vocalization made by rounding and pursing the lips with an increase and then a decrease in frequency and intensity; bark, vocalization made by forcing air through vocal chords from the abdomen, producing a short, rasping, low-frequency sound.

Assessing snake fear. This method has been used previously to detect snake fear (Kalin et al., 2001; Nelson et al., 2003). Subjects were adapted to the Wisconsin General Testing Apparatus (WGTA) test cage for $1 \mathrm{hr}$ on the first day. During the next 3 consecutive days of adaptation, animals were given seven each of the following food items: chocolate chips, small candy-coated chocolate pieces, loop-shaped fruit-flavored cereal, cocktail peanut halves, and raisins. These food items were placed randomly on top of the clear plastic stimulus presentation box $(57.2 \mathrm{~cm}$ wide $\times 22.1 \mathrm{~cm}$ long $\times 6.5 \mathrm{~cm}$ high). Subjects remained in the test environment until either all 35 rewards were consumed or $1 \mathrm{hr}$ had elapsed. If animals failed to retrieve a food, a new food item was substituted the following day. The order of retrieval of each food item was recorded to determine the two most preferred for each subject. These preferred items were used as rewards during subsequent testing.

On day 5 of adaptation, monkeys were presented with their two most preferred foods on top of the stimulus presentation box. This was done by opening the WGTA window for $60 \mathrm{sec}$ ( 24 times, with a $45 \mathrm{sec}$ interval between presentations). Food choices were presented in a random order, with each subject's preferred food randomly alternating between the left and right side of the stimulus presentation box. Each subject was required to choose at least one food item from the top of the stimulus presentation box within the allotted $60 \mathrm{sec}$. This criterion had to be met on at least 20 of the 24 presentations. If necessary, the number of adaptation days was increased until this criterion was achieved.

For testing, subjects were randomly presented with their two most preferred foods placed in the center on the left and right side of the clear plastic stimulus presentation box. The box contained one of four stimuli: (1) nothing (i.e., the box was empty), (2) tape (an 8.8-cm-diameter roll of blue masking tape), (3) fake snake (a curled black rubber snake $120 \mathrm{~cm}$ in length), and (4) live snake [a northern pine snake (Pithucus melanoleucusi)]. Each stimulus was presented six times during the test in a pseudorandom order. The real snake stimulus was never presented during the first five trials of the first testing day, and no item from either the snake or the nonsnake stimulus category was presented for more than three consecutive trials. The tape and rubber snake were always positioned in the same location inside the box; the live snake was free to move. Each monkey received the same pseudo-random order of stimuli. Each trial lasted $60 \mathrm{sec}$, regardless of the subject's response, and the intertrial interval was $45 \mathrm{sec}$. Latency for the animal's first treat retrieval was used for analysis.

Hormonal sampling and stress exposure. Basal hormonal status and response to stress were evaluated for each animal twice, at least 1 week apart. This was accomplished by obtaining a $7 \mathrm{ml}$ blood sample immediately before and after $30 \mathrm{~min}$ of stress exposure, which consisted of 10 min of restraint followed by $20 \mathrm{~min}$ of confinement in a transport cage. All samples were collected between 8:00 and 9:30 A.M. to control for circadian variability of the hormones. CSF was immediately collected after the second stress blood sample was obtained. After administration of ketamine $\mathrm{HCl}$ ( $15 \mathrm{mg} / \mathrm{kg}$, i.m.), $3 \mathrm{ml}$ of CSF were obtained by percutaneously puncturing the cisterna magna. CSF samples were immediately frozen on dry ice, maintained at $-70^{\circ} \mathrm{C}$, and then analyzed for concentration of CRF.

Cortisol, ACTH, and CRF measurement. Plasma was immediately separated from whole blood by centrifugation at $4^{\circ} \mathrm{C}$ and frozen at $-70^{\circ} \mathrm{C}$ until assayed. Cortisol was measured in plasma samples using an enzyme immunoassay kit (Diagnostic Systems Laboratories, Webster, TX). The intra-assay coefficient of variation $(\mathrm{CV}) \%$ is $6.1 \%$, and the interassay CV\% is $6.3 \%$. The detection limit for this assay is $0.125 \mathrm{ng}$. ACTH was measured using a radioimmunoassay (RIA; Nichols Institute Diagnostics, San Clemente, CA). The intra-assay CV\% is $2.2 \%$, and the interassay $\mathrm{CV} \%$ is $7.2 \%$. The detection limit of the assay is $1.0 \mathrm{pg}$.

Assays for CRF were accomplished by RIA using an antiserum (IgG Corp., Nashville, TN) developed in rabbits against CRF coupled to human $\gamma$-globulins with bisdiazotized benzidine. The antiserum is directed against the $\mathrm{N}$-terminal portion of the intact peptide and has the following molar cross-reactivities: hCRF[1-41], 100\%; hCRF[Met(o)21,38], 100\%; hCRF[6-33], 1\%; hCRF[21-41], 0.1\%; hCRF[28-41], <0.1\%; and hCRF[38-41], $<0.1 \%$. It does not cross-react with ACTH, $\alpha$-MSH, $\beta$-lipotropin, $\gamma$-lipotropin, or $\beta$-endorphin. The detection limit was $0.89 \mathrm{pg}$. The intra-assay and interassay CV\% were 6.1 and $10.2 \%$.

Frontal brain electrical asymmetry assessed with EEG. While the animals were manually restrained, $10 \mathrm{~mm}$ gold disk electrodes for recording EEG were attached to the central, left frontal, and right frontal sites, as well as left and right parietal sites according to the international 10/20 system. Additional electrodes were placed on the left and right mastoids (A1, A2). All EEG electrodes and A2 were recorded with reference to A1, although EEG signals were analyzed after being rereferenced to a computed averaged mastoid value. All electrode impedances were $<5 \mathrm{k} \Omega$. EEG was amplified using bioamplifiers (James Long Co., Caroga Lake, NY).

Electrodes were attached to the shaved skin after cleaning and abrading with a mild abrasive skin-prepping paste. A drop of conductive cream was added to the electrodes, and this cream also held the electrodes in place. All EEG measures were recorded and stored digitally according to methods described previously (Davidson et al., 1992).

Twenty-five and $50 \mathrm{mV}$ sine waves were digitized on each channel both before and after data acquisition to calibrate the recorded EEG. Power spectral analysis was performed using Welch's method (Welch, 1967) on all of the data selected in overlapping (50\%) 1 sec chunks that were passed through a Hamming window to minimize end effects. Spectral power estimates $\left(\mu \mathrm{V}^{2} / \mathrm{Hz}\right)$ for the five bands $(1-4,4-8,8-12$, and $13-30 \mathrm{~Hz}$ ) were averaged across chunks within stages of vigilance. The $4-8 \mathrm{~Hz}$ band was chosen because robust lateralized changes have been seen in rhesus monkeys given diazepam (Davidson et al., 1992). Power density measurements were normalized by log transformation. The direction and magnitude of asymmetry were expressed as the logtransformed power density of an electrode position on the right side of the head minus the log-transformed power density of the corresponding electrode on the left side of the head. Positive asymmetry scores mean greater left-sided activation. ANOVAs were performed on frontal and parietal asymmetry scores, comparing different treatments.

Statistical analysis. Repeated-measures ANOVA was used to compare the two CeA-lesioned and control groups. All post hoc comparisons were made using Duncan multiple range tests. Data that were not normally distributed were transformed by using a square root transformation for frequency data and a $\log (X+1)$ transformation for duration data.

\section{Results}

\section{Extent of the lesions in the CeA region}

Figure 1, $a$ and $b$, displays thionine- and GFAP-stained sections from one of the lesioned animals. On the basis of lesion size and the extent to which the lesions were unilateral or bilateral, the animals were divided into a bilateral (Fig. $2 a$ ) and an asymmetric, predominantly unilateral (Fig. $2 b$ ) group. Nine animals were placed in the bilateral group, having bilateral CeA destruction ranging from 46 to $98 \%$ with a mean of $68.7 \%$ total damage. Five animals constituted the asymmetric group. These animals had a mean of $63.8 \%$ destruction on the most affected side (range, 42-86.5\%) with at least two times as much destruction on the most compared with the least affected side. The mean volume of bilateral CeA damage in the asymmetric group was $36.7 \%$. Two of the operated animals had minimal amounts of CeA damage on either side and therefore were dropped from the analyses.

All 14 animals had some damage that was collateral to the 
a

\section{CeA}
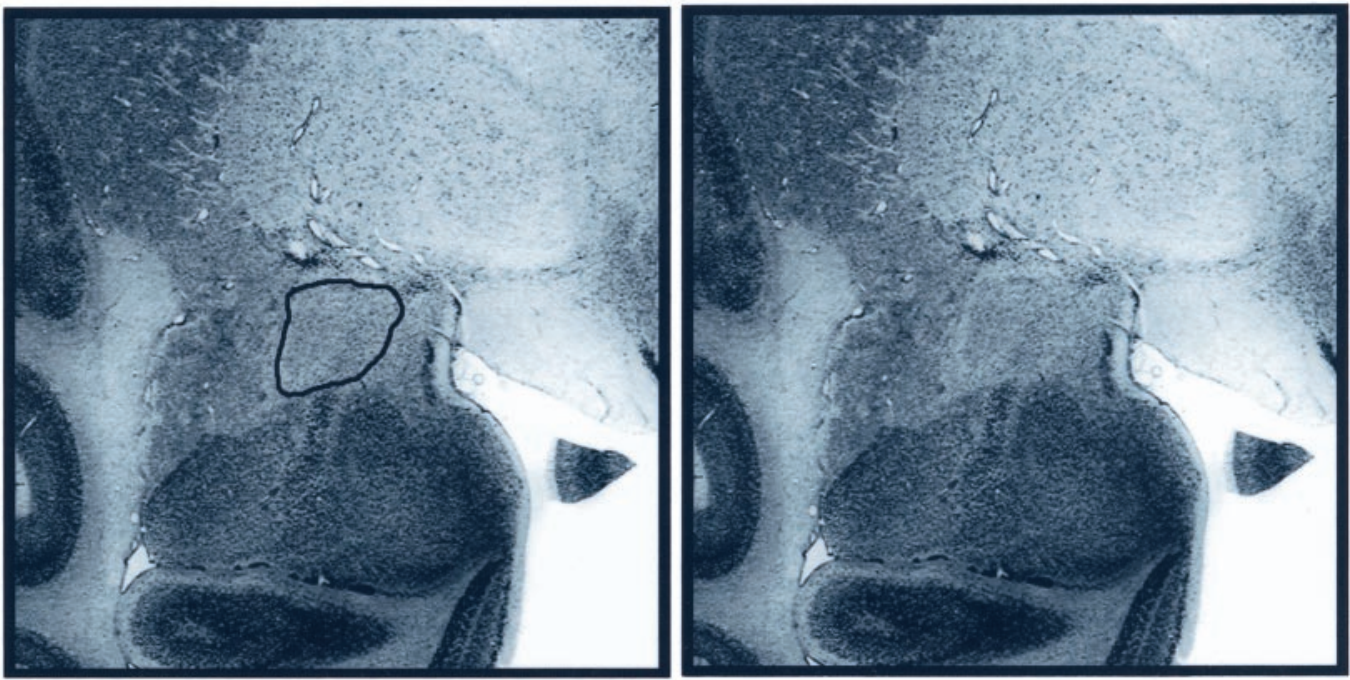

b

Intact
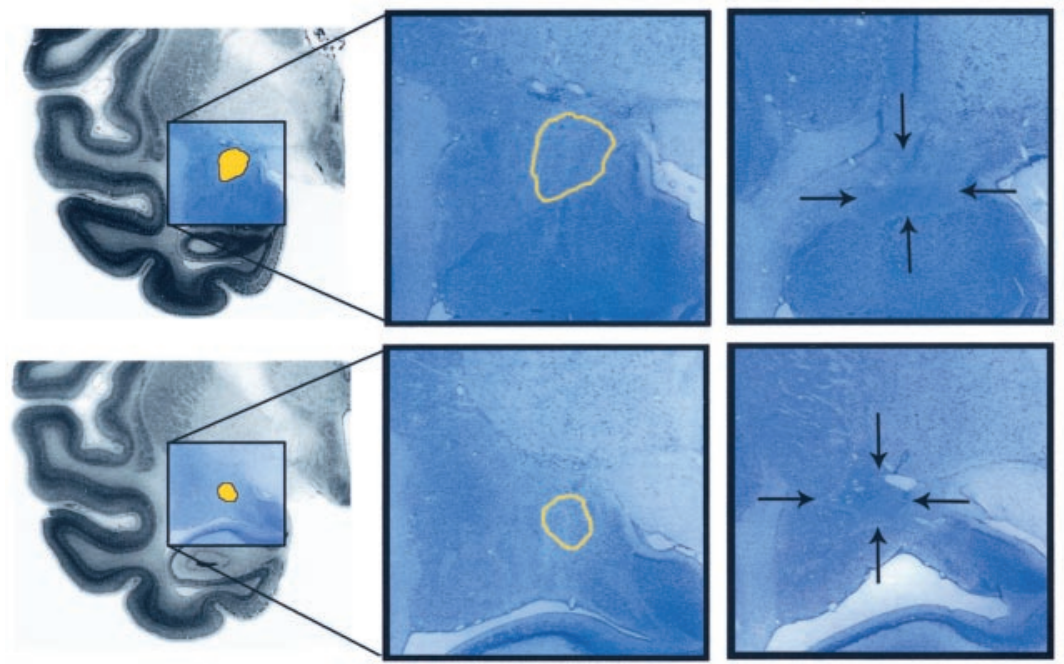

Intact
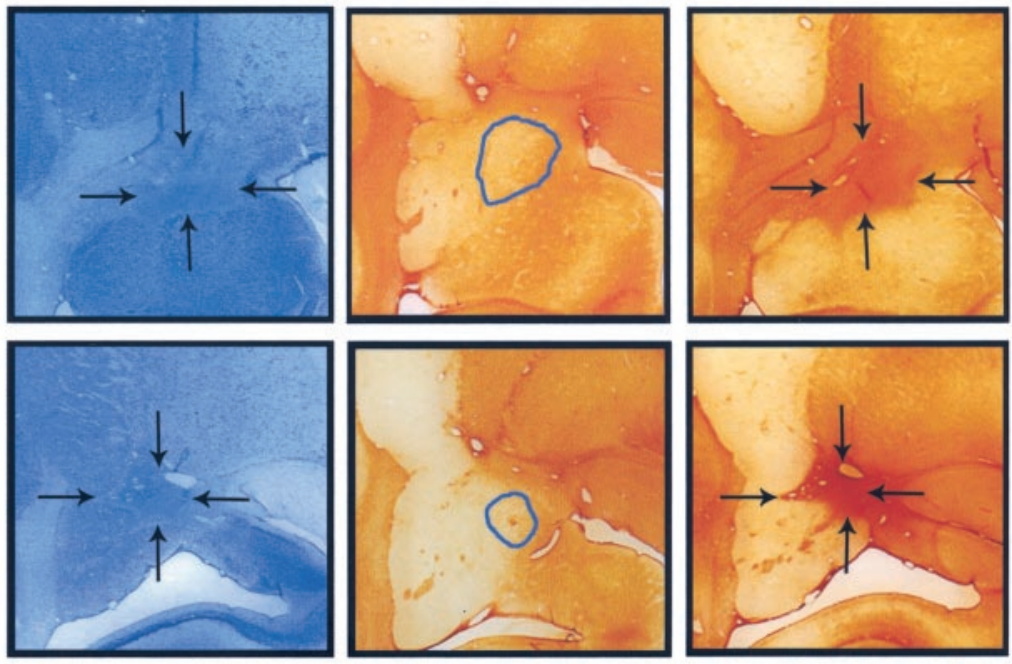

\section{Lesion}
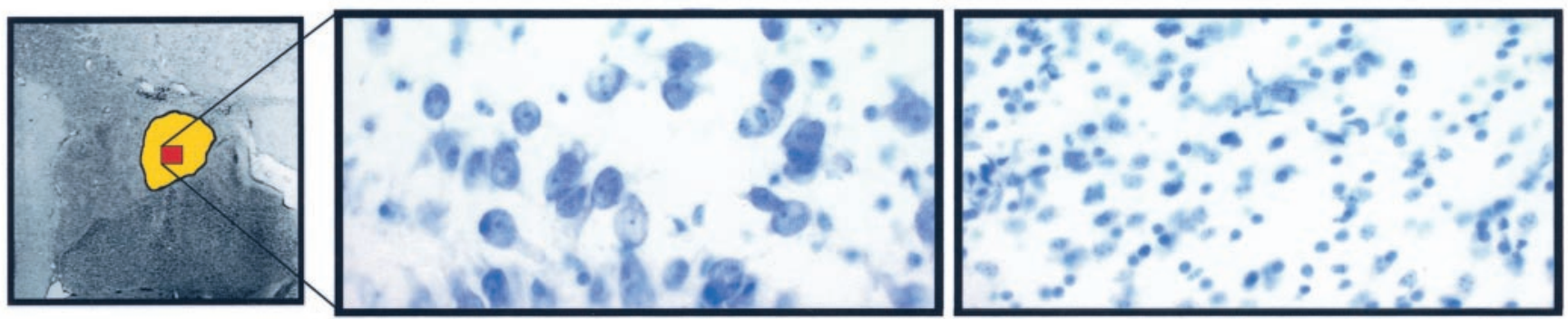

Figure 1. a, Coronal sections through the amygdala demonstrating the boundaries of the CeA. The CeA is located in the dorsal amygdala and is outlined to the left. $b$, Coronal sections from an intact and an ibotenic acid-lesioned animal demonstrate the boundaries of the CeA and the extent of a lesion. The top row displays coronal sections from the left hemisphere through the middle of the amygdala. The hemisected brain at the far left shows the region, in blue, from which the magnified coronal sections were taken. The CeA is depicted in yellow. The first two magnified coronal sections are Nissl stained, and the last two sections are stained with GFAP. The CeA is outlined in the intact sections, whereas in the lesioned sections, arrows indicate the boundaries of the lesion. The middle row displays comparable sections through the posterior amygdala. The bottom row of the figure shows the neuronal loss and glial infiltration in the lesioned compared with the intact CeA (400× magnification). At the far left, the CeA is depicted in yellow, and the area from which the magnified sections were selected is displayed in red. 


\section{Bilateral CeA Lesions}
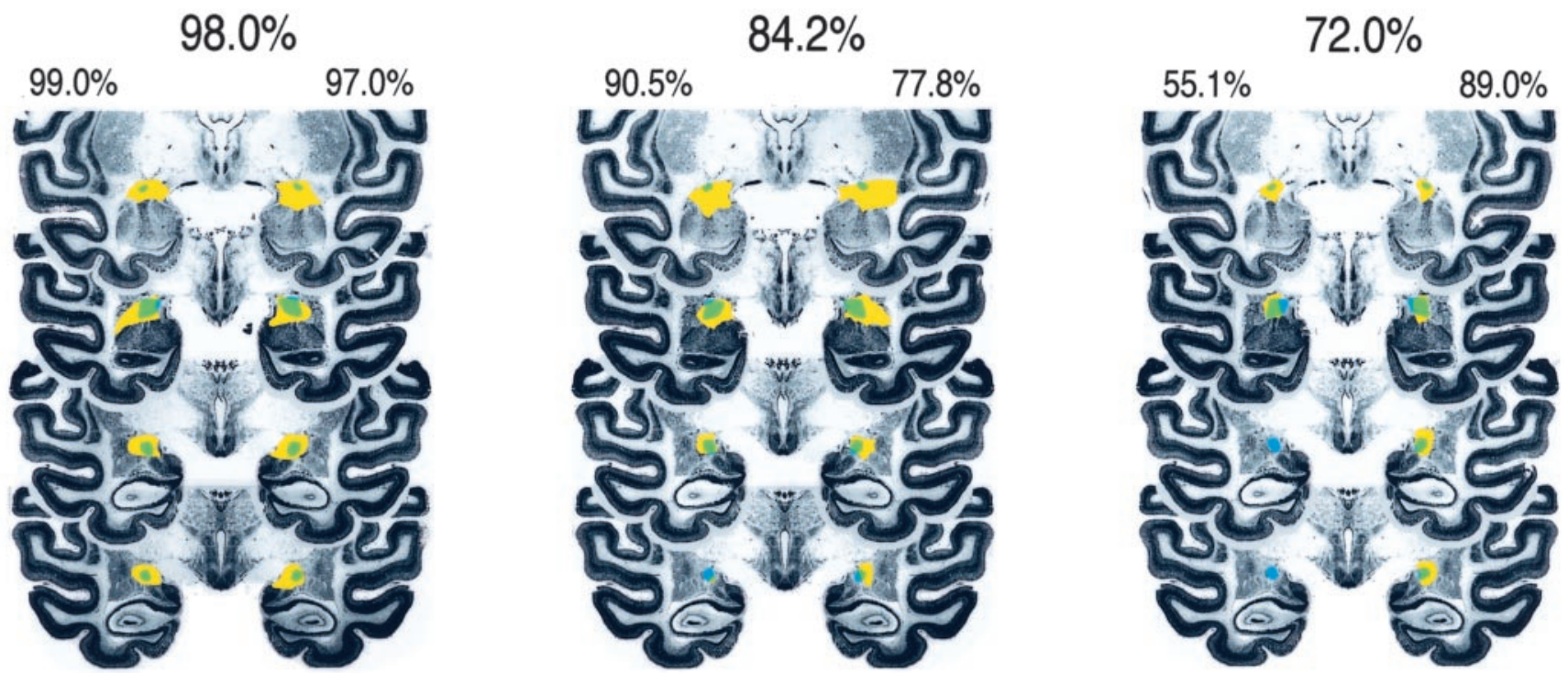

$68.1 \%$
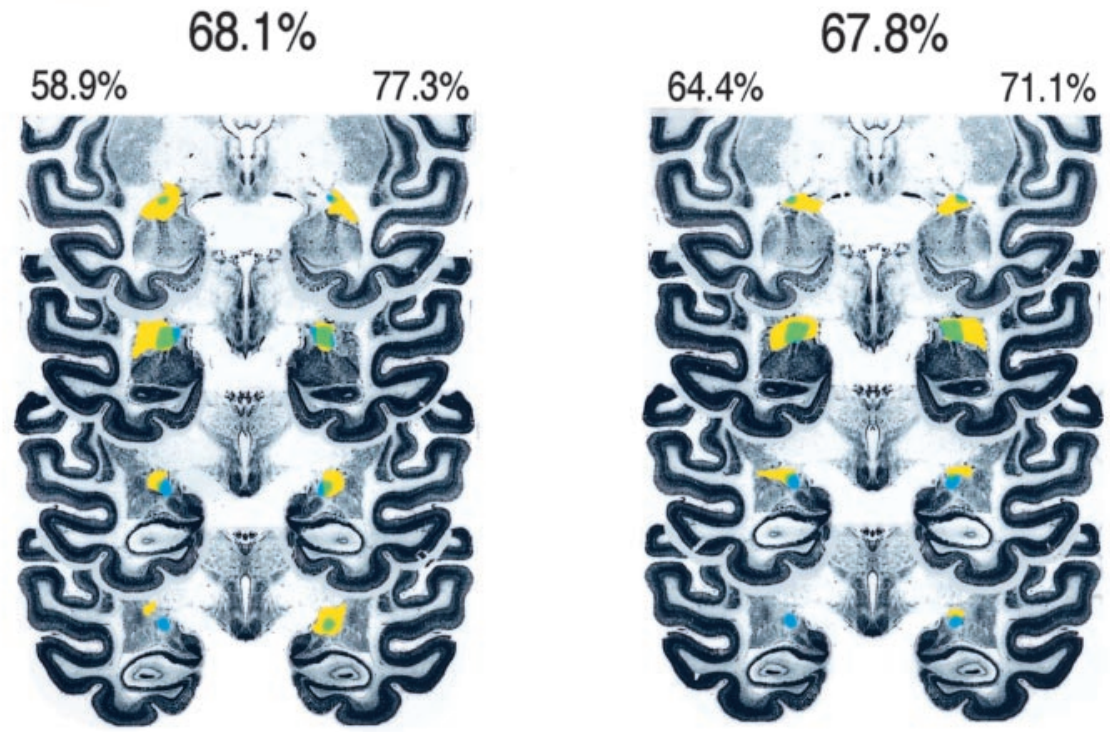

Figure 2. Four coronal sections through the anterior to posterior extent of the CeA are displayed showing the amount of CeA damage in bilateral-lesion ( $a$ ) and asymmetric-lesion ( $b$ ) groups. The intact CeA is depicted in blue, the area of the total lesion is displayed in yellow, and the CeA region that is lesioned is depicted in green. These sections are a subset of the 14 coronal slices that contain the $\mathrm{CeA}$ and that were used to define the percentage of $\mathrm{CeA}$ damage. The percentage destruction of $\mathrm{CeA}$ in each hemisphere and the total amount of CeA destruction are noted for each animal

CeA. Very limited amounts of damage occurred in the lateral, basal, and accessory basal nuclei, and almost no damage was found posterior to the CeA. The range and mean amount of damage for lateral, basal, and accessory basal nuclei were as follows: lateral (mean, $10.4 \%$; range, $0-30 \%$ ), basal (mean, $14.6 \%$; range, $0-50 \%$ ), and accessory basal (mean, 3.9\%; range, $0-10 \%$ ). However, greater amounts of damage occurred in the basal forebrain (substantia innominata and nucleus basalis of Meynert) and the medial amygdala nuclei (medial and cortical). As shown in Figure 2, $a$ and $b$, and as described in Table 1, three of nine animals in the bilateral CeA group had $>25 \%$ bilateral damage to the medial nuclei, and seven of nine animals in this group had $>25 \%$ bilateral damage to the basal forebrain. Four of these animals had $>50 \%$ bilateral damage to the basal forebrain. In the five asymmetric CeA-lesioned animals, two of five animals had
$>25 \%$ unilateral damage to the medial nuclei, and four of five animals had $>25 \%$ damage to the basal forebrain. In two of these animals, the damage was bilateral. Correlational analyses were performed within animals to assess the relationships among amounts of damage in the CeA, basal forebrain, and medial nuclei. Results revealed that $\mathrm{CeA}$ and basal forebrain damage was significantly correlated $(r=0.69 ; p<0.005)$, CeA and medial nuclei damage was not significantly correlated $(r=0.30 ; p>0.05)$, and basal forebrain and medial nuclei damage was significantly correlated $(r=0.71 ; p<0.005)$.

\section{Behavioral effects of the lesions}

Threat-related anxiety and defensive behaviors

Using the human intruder paradigm with three different conditions (A, NEC, and ST) that elicit different anxiety-related defen- 
a

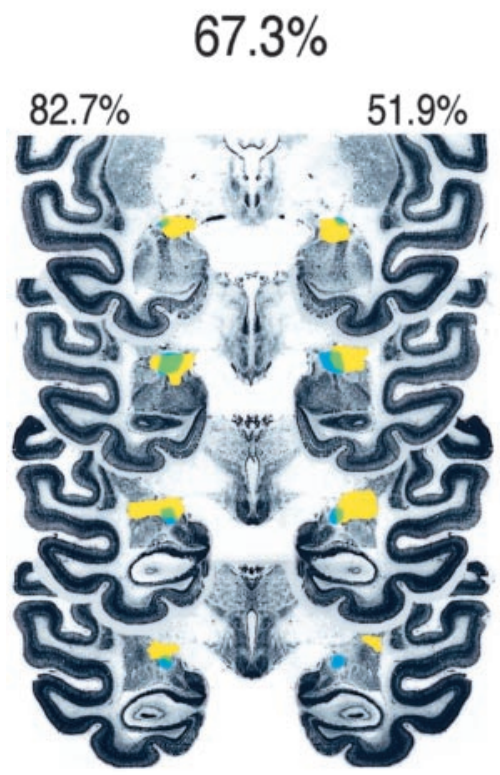

$51.8 \%$

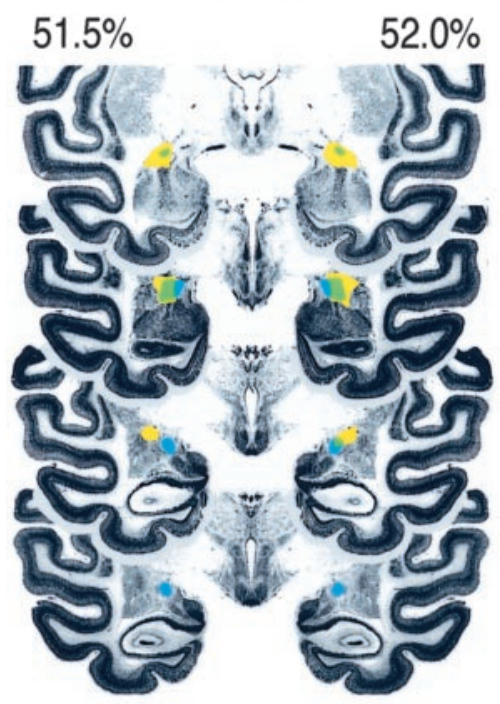

\section{Bilateral CeA Lesions}
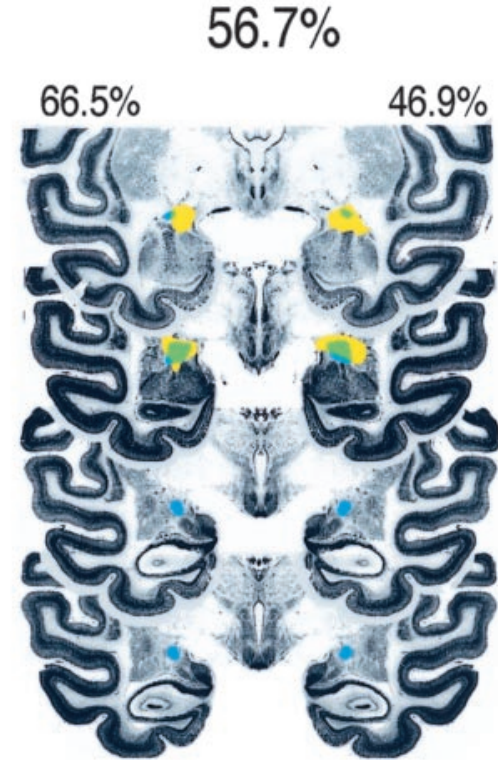

$46.5 \%$

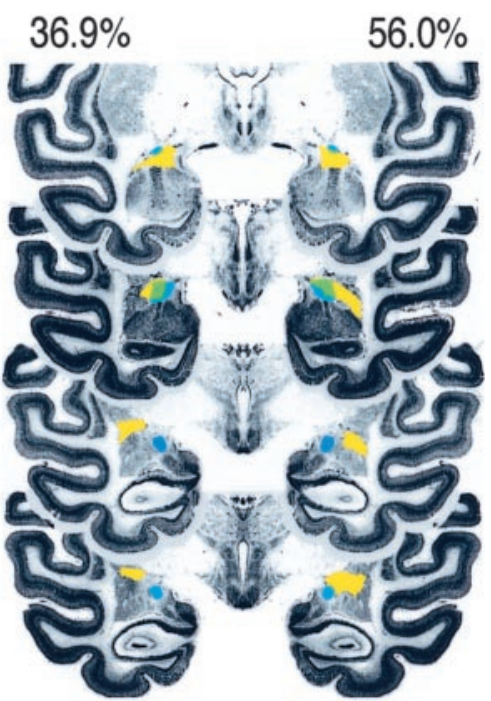

hostility and experimenter orient were analyzed only for the conditions during which the intruder was present (NEC and ST).

The lesions were found to only significantly affect coo vocalizations and freezing duration. Compared with the controls, coos were increased in the bilateral- and asymmetric-lesion groups $(F=3.635$; df 2, $27 ; p<0.04)$. Figure 3 demonstrates that the bilateral-lesion group engaged in significantly less freezing behavior compared with the controls and the asymmetriclesion group $(F=4.330$; df 2,$27 ; p<$ $0.023)$. The mean level of freezing across all conditions was $60.8 \pm 8.96$ and $37.2 \pm 7.87$ $\mathrm{sec}$ in the control and asymmetric groups, respectively compared with $24.2 \pm 4.93 \mathrm{sec}$ in the bilateral group. No significant condition by lesion effects were observed for these behaviors.

\section{Snake fear}

The magnitude of snake fear was assessed using the monkeys' latency to reach for a treat in the presence of the snake, with longer latencies being associated with increased fearfulness. This analysis compared 16 control, 8 bilateral, and 5 asymmetric animals. Only eight animals were in the bilateral group, because one animal was killed before snake-fear testing to verify the lesioning method. Using a between-groups (control, bilateral, and asymmetric lesion) ANOVA with object (snake, fake snake, tape, and nothing) and trial (1-6) as the repeated measures, a main effect of object was observed, such that across all groups the longest latency to reach occurred in the real-snake condition followed by the fakesnake condition, the tape condition, and the nothing condition $(F=21.41$; df 3,78 ; $p<0.0001)$. Figure 4 demonstrates a significant object by lesion interaction $(F=2.88$; df 6,$78 ; p<0.014)$. The latencies to reach in the real-snake condition were significantly less in the bilateral compared with the control and asymmetric groups. In the fake-snake condition, the latency to reach

sive responses, we examined the effects of the lesions on coo and bark vocalizations, freezing behavior, experimenter orient, and defensive hostility. These behaviors were selected because they are the predominant adaptive responses that occur in the A (coo), NEC (freezing, experimenter orient), and ST (bark, defensive hostility) conditions. Using a between-group (control, asymmetric, and bilateral lesion) ANOVA with condition (A, NEC, and ST) as the repeated measure, we found significant main effects for condition for all of these behaviors. As expected, compared with the other conditions, significantly more coos occurred during A $(F=9.751 ;$ df 2,$54 ; p<0.0002)$ and significantly more barks occurred during ST $(F=11.697 ; \mathrm{df} 2,54 ; p<0.0001)$. The NEC condition was associated with significantly more freezing $(F=$ 64.024; df 2, 54; $p<0.0001)$ and experimenter orient $(F=16.90$; df 1,$27 ; p<0.0003)$. Significantly more defensive hostility occurred during ST $(F=94.49$; df 1,$27 ; p<0.0001)$. Defensive for the bilateral lesion group was significantly less than that for the control group. In contrast to the control and asymmetric lesion groups, in the bilateral lesion group the latency to reach during the snake conditions was not significantly greater than the tape and nothing conditions. The latency to reach in the presence of the tape and when no object was present did not significantly differ among the groups.

Effects of the lesions on the pituitary-adrenal system, CSF CRF, and EEG asymmetry

Using a between-groups (control, bilateral, and asymmetric lesion) ANOVA with test ( 1 and 2) and condition (baseline, stress) as the repeated measures, main effects of stress were observed for plasma cortisol $(F=311.95$; df 1,$27 ; p<0.0001)$ and ACTH concentrations $(F=76.20$; df 1,$27 ; p<0.0001)$, with stress 


\section{Asymmetric CeA Lesions}

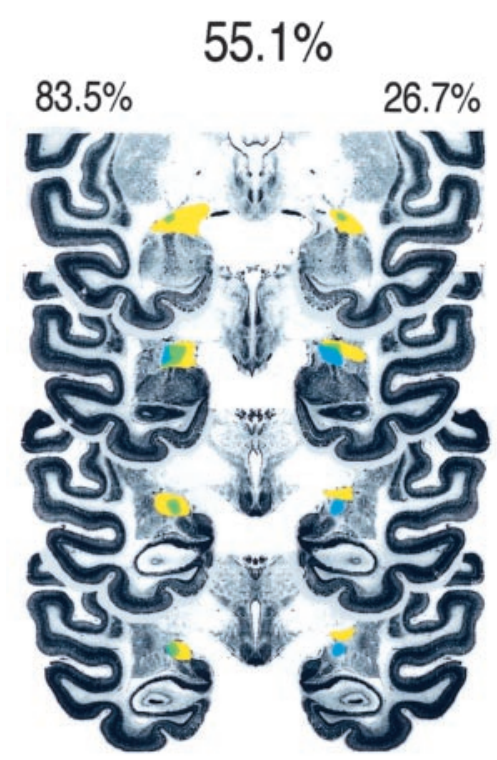

$47.3 \%$

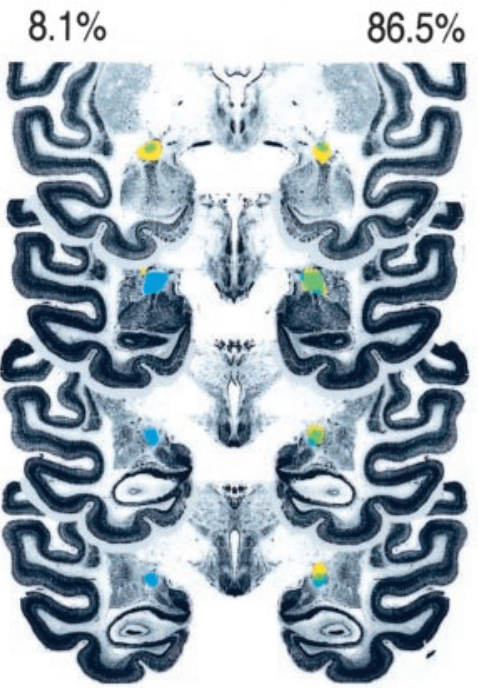

$36.8 \%$

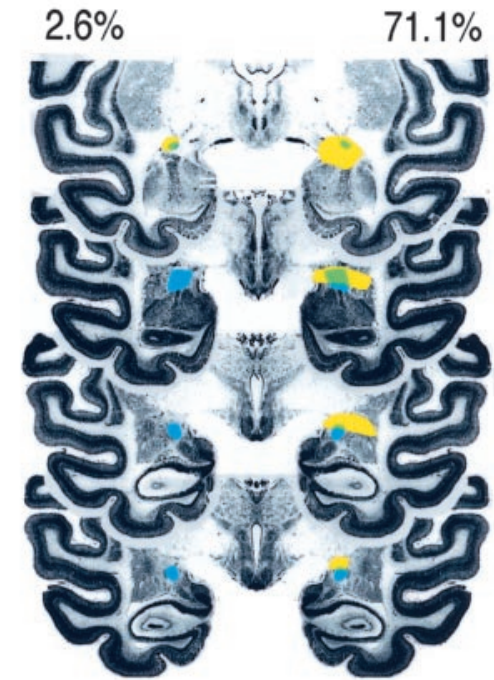

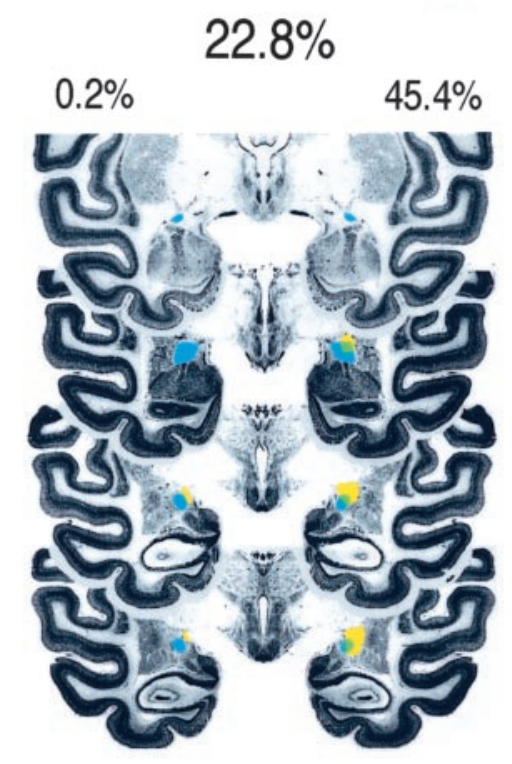

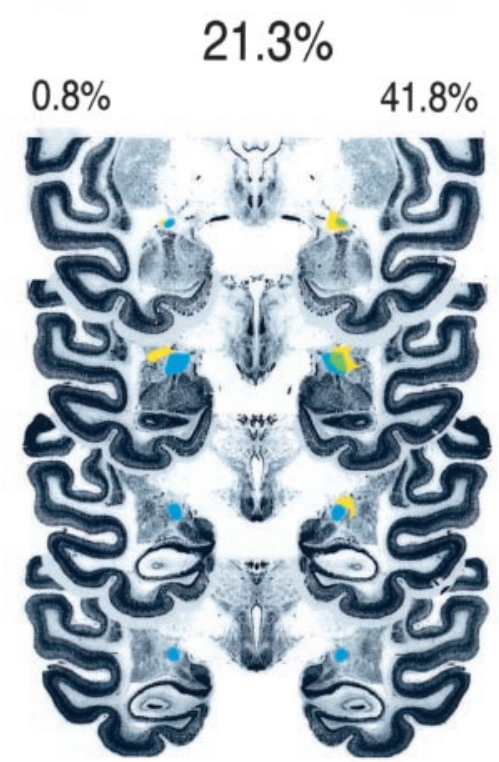

Figure 2. Continued.

inducing large increases in the concentrations of these hormones. Although no lesion or stress by lesion interactions were found for cortisol, a regression analysis revealed that the mean amount of amygdala damage was negatively correlated with baseline $(r=$ $-0.55 ; p<0.025)$ and stress-induced $(r=-0.58 ; p<0.025)$ cortisol concentrations in the 14 lesioned animals. Analysis of the ACTH data revealed a main effect of lesion and no stress by lesion interaction. Figure $5 a$ demonstrates that compared with controls, ACTH concentrations were decreased in the bilateraland asymmetric-lesion groups $(F=3.629$; df 2, 27; $p<0.04)$.

A main effect of lesion was also observed for CSF CRF concentrations $(F=3.30$; df 2,$27 ; p=0.052)$. As can be seen in Figure 5b, CSF CRF concentrations were reduced in the bilaterallesion group compared with the asymmetric-lesion and control groups.

ANOVA of the asymmetric frontal EEG activity revealed no significant differences among the groups. The mean asymmetry score for the bilateral group was -0.09 , the asymmetric group was -0.03 , and the control group was $-0.01(F=1.27$; df 2,26; $p=0.297)$.

\section{Discussion}

These findings demonstrate involvement of the primate CeA region in mediating threat-related anxiety and acute fear-related behavioral and hormonal responses. In addition to reducing snake fear and pituitary-adrenal activity, the CeA lesions resulted in decreased expression of threat-induced freezing and reduced CSF CRF concentrations. It appears that some of the characteristics that we previously considered to be part of the anxious endophenotype (Kalin and Shelton, 1989; Kalin et al., 1998, 2000) are modulated by the CeA. Because it was impossible to lesion the CeA without affecting the basal forebrain and medial 


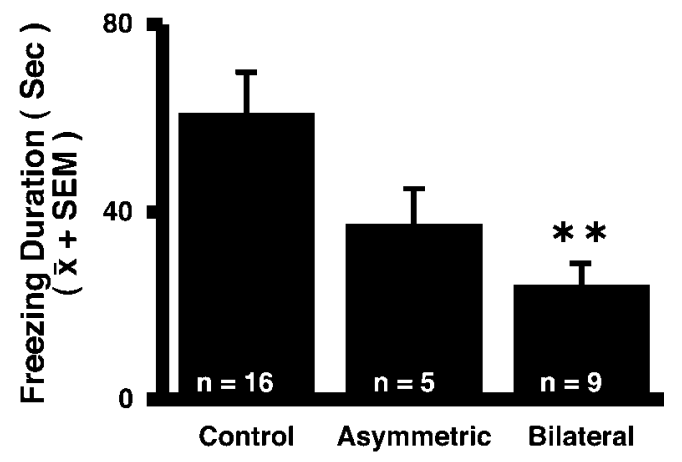

Figure 3. Effects of CeA lesions on freezing duration in the human intruder paradigm. For analysis, the data were transformed to achieve normality $(F=4.330$; $\mathrm{df} 2,27 ; p<0.023)$. Duncan post hoc test: ${ }^{* *} p<0.01$ differs from all groups.

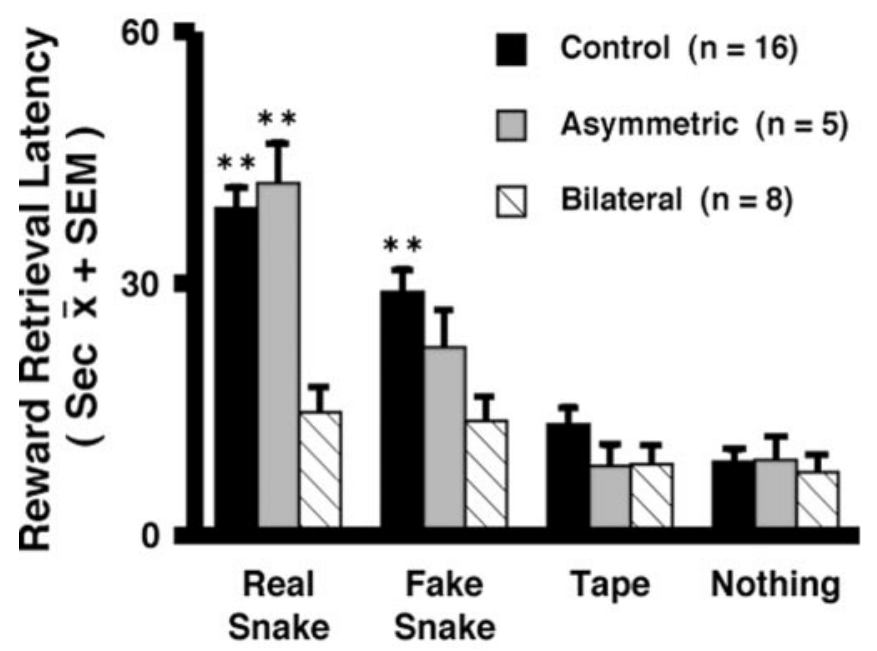

Figure 4. Effects of CeA lesions on snake fear as determined by the monkeys' latency to reach for a treat in the presence of snake stimuli compared with a neutral stimulus and no stimulus. The object by lesion interaction was significant $(F=2.88 ; \mathrm{df} 6,78 ; p<0.014)$. Duncan post hoc test: ${ }^{* *} p<0.01$ differs from bilateral group.

amygdala nuclei, it is feasible that the damage to these regions could be contributing to the effects. Subjects 7 and 8 in the bilateral group had little basal forebrain damage (Table 1). Compared with the other bilaterally lesioned animals with more extensive basal forebrain damage, these two animals had the second and fifth lowest freezing scores and had similarly low fear responses to the snake. This suggests that the blunted fear responses observed in the bilateral animals are likely caused by CeA damage and not damage to the basal forebrain. It is important to keep in mind that the findings from this study were obtained from adolescent monkeys. Although there is no apparent reason to believe that these findings are not generalizable to adult monkeys, the possibility exists and should be considered.

Neuroanatomical studies in rodents and primates demonstrate that the CeA is connected to other key brain regions that allow it to mediate many aspects of the stress response as well as of fear and anxiety (Amaral et al., 1992; Davis, 2000). Within the amygdala, the CeA receives input from the basolateral regions and sends efferents to several subcortical sites involved in mediating the stress response; many of these sites then send projections back to the CeA. These include the ventral tegmental area, locus ceruleus, parabrachial nucleus, trigeminal nucleus, periaqueductal gray, paraventricular nucleus (PVN), lateral hypothala- a

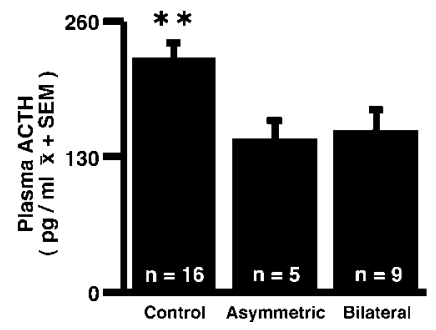

b

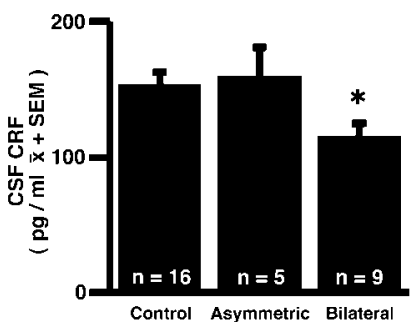

Figure 5. Effects of CeA lesions on plasma ACTH concentrations $(a)(F=3.629 ; \mathrm{df} 2,27 ; p<$ $0.04)$ and CSF CRF concentrations $(b)(F=3.30 ; \mathrm{df} 2,27 ; p=0.052)$. Duncan post hoc test: ${ }^{*} p<0.05$, bilateral group differs from control group; ${ }^{* *} p<0.01$, control differs from other groups.

Table 1. Percentage of destruction of the CeA, medial nuclei (MN), and basal forebrain (BF) for the 14 lesioned animals

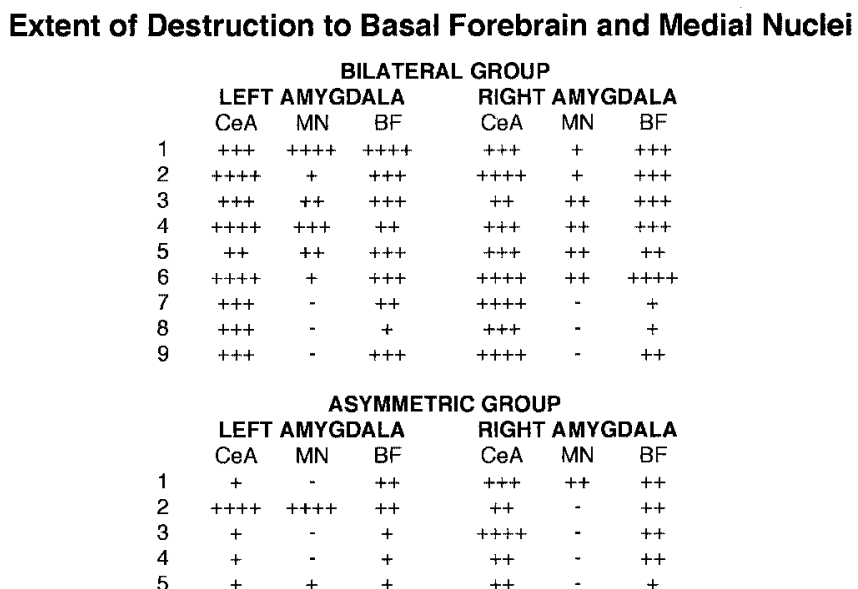

Percentage of destruction for each region is indicated as follows: - , no destruction; + , up to $25 \%$; + +, $26-50 \%$; ,$+++ 51-75 \%$; + + + +, $76-100 \%$.

mus, substantia innominata, bed nucleus of the stria terminalis, and nucleus basalis of Meynert (LeDoux et al., 1988; Amaral et al., 1992; Davis, 2000). Numerous studies in rodents and other subprimate species using CeA lesions and reversible inactivation strategies demonstrate involvement of the CeA in innate and learned behavioral and physiological responses to aversive stimuli (Kapp et al., 1979; Beaulieu et al., 1987; Davis et al., 1994; Campeau and Davis, 1995; Walker and Davis, 1997; Davis, 2000; LeDoux, 2000). Despite the wealth of knowledge from these studies, there are no reports assessing the effects of selective CeA lesions in primates.

In the present study, we found that bilaterally lesioning the CeA region resulted in a reduction in freezing behavior occurring in the human intruder paradigm. This is of interest because freezing is an adaptive defensive response that reflects an underlying state of anxiety, and we have suggested previously that freezing in monkeys is similar to human behavioral inhibition (Kalin and Shelton, 1989). Many (Davis, 2000) but not all (Killcross et al., 1997) studies in rodents also demonstrate that CeA lesions decrease freezing behavior. Also, in a previous study we failed to demonstrate an effect of large, near total, ibotenic acid amygdala lesions on monkeys' freezing behavior (Kalin et al., 2001). The difference between this finding in our initial and current study cannot be explained by differences in the amount of CeA damage, because most of the monkeys in the first study had CeA damage comparable with that of the monkeys in the present study. Also, 
in both studies, there were similar amounts of damage dorsal to the amygdala in basal forebrain structures. One difference between the studies is that in the large lesion study, monkeys were pretested in the human intruder paradigm before surgery. Pretesting did not occur in the present study. In the past, we observed a small reduction in freezing duration from the first to subsequent exposures to the human intruder paradigm. Therefore, it is possible that pre-exposure resulted in some degree of habituation. Because the amygdala robustly responds to novelty (Whalen, 1998; Davis and Whalen, 2001; Schwartz et al., 2003a) and because its activity has been shown to rapidly habituate (Breiter et al., 1996; Fischer et al., 2003), it is possible that the amygdala mediates the novel component of freezing behavior that rapidly habituates. Thus, in the first study, if the novel component of freezing had habituated before lesioning, an effect of the lesion might not have been detected. It is also possible that selective CeA lesions have effects other than those of larger amygdala lesions that, in addition to the CeA, involve the basal, accessory basal, and lateral nuclei. If this were the case, it would suggest that a much more complicated functional relationship exists among the CeA, other amygdala nuclei, and extra-amygdala sites that are involved in mediating freezing behavior.

In addition to freezing behavior, the CeA lesions affected coo calls such that an increase in cooing occurred across all human intruder conditions in both the asymmetric- and bilateral-lesion groups. Coos function as a call for help and occur with greatest frequency in the alone and stare conditions (Kalin and Shelton, 1989). In contrast to the effect on coos, the lesions were without significant effects on bark vocalizations. Barks are aggressive and occur with greatest frequency in the stare condition. The lesions also did not significantly affect the amount of time the monkeys engaged in defensive hostility or orienting behavior directed toward the intruder. However, because of the incomplete nature of the lesions, caution should be used in interpreting these negative findings.

Consistent with our previous large amygdala lesion study and with findings from other laboratories (Meunier et al., 1999; Kalin et al., 2001), we found that bilateral CeA lesions decreased snake fear. Compared with control and asymmetrically lesioned monkeys, monkeys with bilateral CeA lesions demonstrated a decreased latency to reach for a treat in the presence of the real and fake snake. Latencies to reach for the treat in the presence of the roll of tape or in the nothing condition did not significantly differ in monkeys with bilateral lesions compared with controls and asymmetrically lesioned animals.

The data also revealed that the lesions affected the pituitaryadrenal system. Although group effects were not observed for cortisol, there were significant negative correlations between CeA lesion size and basal and stress-induced cortisol concentrations. ACTH concentrations were significantly decreased in both the bilaterally and the asymmetrically lesioned animals. Because ACTH concentrations were reduced across both baseline and stress conditions, it is likely that the lesions resulted in an overall reduction in pituitary-adrenal activity. Studies in rodents also demonstrate that CeA lesions blunt stress-induced ACTH secretion (Beaulieu et al., 1987; Prewitt and Herman, 1994). To be confident in the apparent lack of group effect of the lesions on cortisol concentrations, more frequent blood sampling should be performed, because the pituitary-adrenal system is dynamic and ACTH and cortisol concentrations can rapidly fluctuate.

CRF is a peptide that is found in the PVN of the hypothalamus and plays a major role in regulating activity of the pituitaryadrenal system. CRF is also found in extrahypothalamic brain regions, including the cortex, limbic structures, and brainstem (Sawchenko et al., 1993). In these regions, CRF has been implicated in mediating the behavioral, emotional, and autonomic aspects of the stress response. A wealth of preclinical data demonstrate that CRF is anxiogenic and that some of its effects are mediated in the amygdala (Bakshi et al., 2002). Furthermore, studies from depressed humans demonstrate increased CSF CRF concentrations, implicating overactivity of the CRF system in mediating affective psychopathology (Arborelius et al., 1999). Studies in nonhuman primates demonstrate that higher levels of CSF CRF are associated with increased anxiety, as indexed by increased freezing behavior (Kalin et al., 2000), and we have suggested previously that increased CSF CRF concentrations occur as part of the anxious endophenotype (Kalin et al., 2000). In the present study, we found that the bilateral lesions resulted in decreased CSF CRF concentrations. The dynamics affecting CSF CRF concentrations have been studied extensively. Work from our laboratory, in monkeys, suggested that the origin of CRF in CSF is not from the PVN but rather from other extrahypothalamic sites (Kalin et al., 1987). Because CRF is found in the CeA, the possibility exists that the reduced CSF concentrations occurring in the bilateral-lesion group are directly attributable to lesion-induced death of CRF-containing neurons. Alternatively, overall reductions in anxiety and decreased activation of CeA projection sites containing CRF could account for the finding.

Finally, consistent with our previous large amygdala lesion study, the CeA lesions were without significant effect on patterns of asymmetric frontal brain electrical activity (Kalin et al., 2001). Because asymmetric right frontal activity is associated with negative affect and increased cortisol in humans (Davidson et al., 2000; Buss et al., 2003) and increased anxiety-related responses, increased pituitary-adrenal activity, and increased CSF CRF concentrations in monkeys (Kalin et al., 1998, 2000), it might be expected that amygdala lesions would shift the activity from the right to the left hemisphere. Furthermore, we demonstrated previously in monkeys that administration of the anxiolytic agent diazepam results in a shift in activity from right to left frontal asymmetry (Davidson et al., 1992). The finding of a lack of effect of the lesions suggests that this electrophysiological indicator of prefrontal activity and emotional disposition is not dependent on amygdala activity. In summary, the present study demonstrates a role for the primate $\mathrm{CeA}$ in mediating anxiety-related defensive responses, acute fear responses, pituitary-adrenal activity, and brain CRF systems. Overactivity of the amygdala has been hypothesized to play a role in mediating the pathophysiology of some affective and anxiety disorders (Drevets and Raichle, 1995; Davidson, 2002; Schwartz et al., 2003b) and as a mechanism underlying extreme behavioral inhibition in children (Schwartz et al., 2003b) that is a risk factor for the later development of anxiety disorders. We have suggested that the anxious endophenotype in rhesus monkeys models human anxiety, and in this regard we believe that the data support a mechanistic role for the CeA.

\section{References}

Aggleton JP, Passingham RE (1981) Syndrome produced by lesions of the amygdala in monkeys (Macaca mulatta). J Comp Physiol Psychol 95:961-977.

Amaral DG (2002) The primate amygdala and the neurobiology of social behavior: implications for understanding social anxiety. Biol Psychiatry 51:11-17.

Amaral DG, Price JL (1983) An air pressure system for the injection of tracer substances into the brain. J Neurosci Methods 9:35-43. 
Amaral DG, Price JL, Pitkanen A, Carmichael ST (1992) Anatomical organization of the primate amygdaloid complex. In: The amygdala (Aggleton J, ed), pp 1-67. New York: Wiley.

Arborelius L, Owens MJ, Plotsky PM, Nemeroff CB (1999) The role of corticotropin-releasing factor in depression and anxiety disorders. J Endocrinol 160:1-12.

Bakshi VP, Kalin NH (2000) Corticotropin-releasing hormone and animal models of anxiety: gene-environment interactions. Biol Psychiatry 48:1175-1198.

Bakshi VP, Smith-Roe S, Newman SM, Grigoriadis DE, Kalin NH (2002) Reduction of stress-induced behavior by antagonism of corticotropinreleasing hormone $2\left(\mathrm{CRH}_{2}\right)$ receptors in lateral septum or $\mathrm{CRH}_{1}$ receptors in amygdala. J Neurosci 22:2926-2935.

Beaulieu S, Di Paolo T, Cote J, Barden N (1987) Participation of the central amygdaloid nucleus in the response of adrenocorticotropin secretion to immobilization stress: opposing roles of the noradrenergic and dopaminergic systems. Neuroendocrinology 45:37-46.

Biederman J, Chaloff J, Hirshfeld DR, Kagan J (1993) A 3-year follow-up of children with and without behavioral inhibition. J Am Acad Child Adolesc Psychiatry 32:814-821.

Blanchard DC, Blanchard RJ (1972) Innate and conditioned reactions to threat in rats with amygdaloid lesions. J Comp Physiol Psychol 81:281-290.

Breiter HC, Etcoff NL, Whalen PJ, Kennedy WA, Rauch SL, Buckner RL, Strauss MM, Hyman SE, Rosen BR (1996) Response and habituation of the human amygdala during visual processing of facial expression. Neuron $17: 875-887$.

Buss KA, Schumacher JR, Dolski I, Kalin NH, Goldsmith HH, Davidson RJ (2003) Right frontal brain activity, cortisol, and withdrawal behavior in 6-month-old infants. Behav Neurosci 117:11-20.

Campeau S, Davis M (1995) Involvement of the central nucleus and basolateral complex of the amygdala in fear conditioning measured with fearpotentiated startle in rats trained concurrently with auditory and visual conditioned stimuli. J Neurosci 3:2301-2311.

Davidson RJ (2002) Anxiety and affective style: role of prefrontal cortex and amygdala. Biol Psychiatry 51:68-80.

Davidson RJ, Kalin NH, Shelton SE (1992) Lateralized effects of diazepam on frontal brain electrical asymmetries in rhesus monkeys. Biol Psychiatry $32: 438-451$.

Davidson RJ, Jackson DC, Kalin NH (2000) Emotion, plasticity, context, and regulation: perspectives from affective neuroscience. Psychol Bull 126:890-909.

Davis M (1992) The role of the amygdala in fear and anxiety. Annu Rev Neurosci 15:353-375.

Davis M (2000) The role of the amygdala in conditioned and unconditioned fear and anxiety. In: The amygdala, Ed 2 (Aggleton J, ed), pp 213-287. New York: Oxford.

Davis M, Whalen PJ (2001) The amygdala: vigilance and emotion. Mol Psychiatry $1: 12-34$

Davis M, Hitchock JM, Bowers MB, Berridgy CW, Melia KR, Roth RH (1994) Stress-induced activation of prefrontal cortex dopamine turnover: blockade by lesion of the amygdala. Brain Res 664:207-210.

Drevets WC, Raichle ME (1995) Positron emission tomographic imaging studies of human emotional disorders. In: The cognitive neurosciences (Gazzaniga MS, ed), pp 1153-1164. Cambridge, MA: MIT.

Emery NJ, Capitanio JP, Mason WA, Mendoza SA, Amaral DG (2001) The effects of bilateral lesions of the amygdala on dyadic social interactions in rhesus monkeys (Macaca mulatta). Behav Neurosci 115:515-544.

Fischer H, Wright CI, Whalen PJ, McInerney SC, Shin LM, Rauch SL (2003) Brain habituation during repeated exposure to fearful and neutral faces: a functional MRI study. Brain Res Bull 59:387-392.

Fuster JM (1997) The prefrontal cortex: anatomy, physiology, and neuropsychology of the frontal lobe. Philadelphia: Lippincott-Raven.

Kagan J, Reznick JS, Snidman N (1988) Biological bases of childhood shyness. Science 240:167-171.

Kalin NH (1993) The neurobiology of fear. Sci Am 268:94-101.
Kalin NH, Shelton SE (1989) Defensive behaviors in infant rhesus monkeys: environmental cues and neurochemical regulation. Science 243:1718-1721.

Kalin NH, Shelton SE, Barksdale CM, Brownfield MS (1987) A diurnal rhythm in cerebrospinal fluid corticotropin-releasing hormone different from the rhythm of pituitary-adrenal activity. Brain Res 426:385-391.

Kalin NH, Larson C, Shelton SE, Davidson RJ (1998) Asymmetric frontal brain activity, cortisol, and behavior associated with fearful temperaments in rhesus monkeys. Behav Neurosci 112:286-292.

Kalin NH, Shelton SE, Davidson RJ (2000) Cerebrospinal fluid corticotropin-releasing hormone levels are elevated in monkeys with patterns of brain activity associated with fearful temperament. Biol Psychiatry 47:579-585.

Kalin NH, Shelton SE, Davidson RJ, Kelley AE (2001) The primate amygdala mediates acute fear but not the behavioral and physiological components of anxious temperament. J Neurosci 21:2067-2074.

Kapp BS, Frysinger RC, Gallagher M, Haselton JR (1979) Amygdala central nucleus lesions: effect on heart rate conditioning in the rabbit. Physiol Behav 23:1109-1117.

Killcross S, Robbins TW, Everitt BJ (1997) Different types of fearconditioned behaviour mediated by separate nuclei within amygdala. $\mathrm{Na}-$ ture 388:377-380.

Kling A, Brothers LA (1992) The amygdala and social behavior. In: The amygdala (Aggleton J, ed), pp 353-377. New York: Wiley.

Kluver H, Bucy PC (1939) Preliminary analysis of functions of the temporal lobes in monkeys. AMA Arch Neurol Psychiatry 42:979-1000.

LeDoux JE (2000) Emotion circuits in the brain. Annu Rev Neurosci 23:155-184.

LeDoux JE, Iwata J, Cicchetti P, Reis DJ (1988) Different projections of the central amygdaloid nucleus mediate autonomic and behavioral correlates of conditioned fear. J Neurosci 8:2517-2529.

Meunier M, Bachevalier J, Murray EA, Malkova L, Mishkin M (1999) Effects of aspiration versus neurotoxic lesions of the amygdala on emotional responses in monkeys. Eur J Neurosci 11:4403-4418.

Murray EA, Mishkin M (1998) Object recognition and location memory in monkeys with excitotoxic lesions of the amygdala and hippocampus. J Neurosci 18:6568-6582.

Nelson EE, Shelton SE, Kalin NH (2003) Individual differences in the responses of naive rhesus monkeys to snakes. Emotion 3:3-11.

Prewitt CM, Herman JP (1994) Lesion of the central nucleus of the amygdala decreases CRH mRNA expression and stress-induced ACTH release. Ann NY Acad Sci 746:438-440.

Sawchenko PE, Imaki T, Potter E, Kovacs K, Imaki J, Vale W (1993) The functional neuroanatomy of corticotrophin-releasing factor. Ciba Found Symp 172:5-21.

Schwartz CE, Wright CI, Shin LM, Kagan J, Whalen PJ, McMullin KG, Rauch SL (2003a) Differential amygdalar response to novel versus familiar neutral faces: a functional MRI probe developed for studying inhibited temperament. Biol Psychiatry 53:854-862.

Schwartz CE, Wright CI, Shin LM, Kagan J, Rauch SL (2003b) Inhibited and uninhibited infants "grown up": adult amygdalar response to novelty. Science 300:1952-1953.

Walker DL, Davis M (1997) Double dissociation between the involvement of the bed nucleus of the stria terminalis and the central nucleus of the amygdala in startle increases produced by conditioned versus unconditioned fear. J Neurosci 17:9375-9383.

Weiskrantz L (1956) Behavioral changes associated with ablation of the amygdaloid complex in monkeys. J Comp Physiol Psychol 4:381-391.

Welch PD (1967) The use of fast Fourier transform for the estimation of power spectra: a method based on time averaging over short, modified periodograms. IEEE Trans Audio Electroacoust Av 15:70-73.

Whalen PJ (1998) Fear, vigilance, and ambiguity: initial neuroimaging studies of the human amygdala. Curr Dir Psychol Sci 7:177-188.

Zola-Morgan S, Squire LR, Alvarez-Royo P, Clower RP (1991) Independence of memory functions and emotional behavior: separate contributions of the hippocampal formation and the amygdala. Hippocampus $1: 207-220$. 